\title{
The enzymatic activity of Arabidopsis protein arginine methyltransferase 10 is essential for flowering time regulation
}

\author{
Lifang Niu ${ }^{1,2,4^{*}}$, Falong $\mathrm{Lu}^{1,3,4^{*}}$, Taolan Zhao ${ }^{1,4^{*}}$, Chunyan Liu ${ }^{1}$, Xiaofeng Cao ${ }^{1 凶}$ \\ ${ }^{1}$ State Key Laboratory of Plant Genomics and National Center for Plant Gene Research, Institute of Genetics and \\ Developmental Biology, Chinese Academy of Sciences, Beijing 100101, China \\ 2 Institute for Agricultural Biosciences, Oklahoma State University, Ardmore, OK 73401, USA \\ ${ }^{3}$ Lineberger Comprehensive Cancer Center, University of North Carolina at Chapel Hill, Chapel Hill, NC 27599, USA \\ ${ }^{4}$ Graduate School of the Chinese Academy of Sciences, Beijing 100039, China \\ $\square$ Correspondence: xfcao@genetics.ac.cn \\ Received April 12, 2012 Accepted May 21, 2012
}

\section{ABSTRACT}

Arabidopsis AtPRMT10 is a plant-specific type I protein arginine methyltransferase that can asymmetrically dimethylate arginine 3 of histone $\mathrm{H} 4$ with auto-methylation activity. Mutations of AtPRMT10 derepress FLOWERING LOCUS $C(F L C)$ expression resulting in a late-flowering phenotype. Here, to further investigate the biochemical characteristics of AtPRMT10, we analyzed a series of mutated forms of the AtPRMT10 protein. We demonstrate that the conserved "VLD" residues and "double-E loop" are essential for enzymatic activity of AtPRMT10. In addition, we show that Arg54 and Cys259 of AtPRMT10, two residues unreported in animals, are also important for its enzymatic activity. We find that Arg13 of AtPRMT10 is the auto-methylation site. However, substitution of Arg13 to Lys13 does not affect its enzymatic activity. In vivo complementation assays reveal that plants expressing AtPRMT10 with VLD-AAA, E143Q or E152Q mutations retain high levels of $F L C$ expression and fail to rescue the late-flowering phenotype of atprmt10 plants. Taken together, we conclude that the methyltransferase activity of AtPRMT10 is essential for repressing $F L C$ expression and promoting flowering in Arabidopsis.

KEYWORDS protein arginine methyltransferases (PRMTs), flowering, methyltransferase activity

\section{INTRODUCTION}

Protein arginine methyltransferases (PRMTs) modify target arginine residues in histones and other substrates to regulate multiple biological processes, including transcriptional regulation, RNA metabolism, protein localization, DNA repair, and signal transduction (Bedford and Clarke, 2009; Wolf, 2009; Ahmad and Cao, 2012). PRMTs are classified into four types depending on their products (Bedford and Clarke, 2009). Type I and type II PRMTs are the major PRMTs that catalyze the formation of omega- $\mathrm{N}^{G}$-monomethylarginine (MMA). Further, type I enzymes yield asymmetric omega- $N^{G}, N^{G}$ dimethylarginine and type II enzymes form symmetric omega- $\mathrm{N}^{\mathrm{G}}, \mathrm{N}^{\prime \mathrm{G}}$-dimethylarginine (SDMA) (Bedford and Clarke, 2009).

PRMTs have the conserved I, post-I, II, and III motifs of the S-adenosyl-L-methionine (SAM)-dependent methyltransferases as well as the unique "double-E" and "THW" motifs of the PRMT subfamily (Schluckebire et al., 1995; Kwak et al., 2003; Cheng et al., 2005). Changing "VLD", the first three highly conserved residues of motif I, to three alanines (AAA) abolishes the in vitro enzymatic activity of PRMT1 and coactivator-associated arginine methyltransferase 1 CARM1/ PRMT4 (Lee et al., 2002; Wada et al., 2002), and affects nucleocytoplasmic shuttling of PRMT1 variant 2 (PRMT1v2) in vivo (Herrmann and Fackelmayer, 2009). The "double-E loop" was named after the two invariant glutamates within the active site of PRMTs (Zhang et al., 2000; Zhang and Cheng, 2003). Crystal structures of PRMTs indicate that the PRMT

\footnotetext{
*These authors contributed equally to the work.
} 
active site is encircled by the SAM-binding domain and the $\beta$ barrel domain. The "double-E loop" forms a hairpin structure which is essential for active site formation (Zhang et al., 2000; Zhang and Cheng, 2003). Site-specific mutation of either glutamate composing the "double-E loop" causes severe reduction or complete abolition of the methyltransferase activity of PRMT1 (Zhang and Cheng, 2003).

PRMTs methylate a wide variety of histone and nonhistone substrates, and some PRMTs including PRMT1, CARM1/PRMT4, PRMT6 and PRMT8 also have auto-methylation activities (Frankel et al., 2002; Sayegh et al., 2007; Kuhn et al., 2009; Gui et al., 2011; Kuhn et al., 2011). The automethylation sites of CARM1s in different species were mapped by mass spectrometry. Amino-acid substitutions in these auto-methylation sites seem to have no effect on CARM1 enzymatic activity (Kuhn et al., 2009, 2011). Nevertheless, the loss of auto-methylation was reported to impair the role of CARM1 in transcriptional activation and alternative splicing, which indicates the importance of auto-methylation in PRMT's biological function (Kuhn et al., 2011).

Nine members of the PRMT family have been identified in Arabidopsis thaliana, and are termed Arabidopsis thaliana protein arginine methyltransferases (AtPRMTs) (Niu et al., 2007; Liu et al., 2010; Ahmad and Cao, 2012). Among them, AtPRMT1a and AtPRMT1b are type I PRMTs, which can asymmetrically methylate histone $\mathrm{H} 4$ at $\mathrm{Arg} 3(\mathrm{H} 4 \mathrm{R} 3)$ and Arabidopsis thaliana fibrillarin 2 (AtFib2) (Niu et al., 2007; Yan et al., 2007). AtPRMT4a and AtPRMT4b are also type I PRMTs which can asymmetrically methylate histone $\mathrm{H} 3$ at Arg 17 (H3R17) (Niu et al., 2008). AtPRMT5 is a type II PRMT which can symmetrically methylate histone H4R3 and non-histone proteins involved in RNA processing (Pei et al., 2007; Wang et al., 2007; Schmitz et al., 2008; Deng et al., 2010; Hong et al., 2010; Sanchez et al., 2010; Zhang et al., 2011b). AtPRMT10 is a plant-specific type I PRMT that asymmetrically di-methylates histone $\mathrm{H} 4 \mathrm{R} 3$ and $\mathrm{H} 2 \mathrm{~A}$ in vitro (Niu et al., 2007). Although AtPRMT10 is of low phylogenetic similarity to other animal PRMTs, it retains all the conserved motifs of PRMTs. Crystal structure of AtPRMT10 indicates that AtPRMT10 adopts a unique conformation compared to other PRMTs with known structure (Cheng et al., 2011).

Flowering as a result of switching from vegetative to reproductive phase is critical for sexual reproduction in higher plants. The crucial decision of when to flower is executed by a complicated network involving endogenous signals and multiple environmental stimuli such as day length, light quality, temperature and nutrition (Amasino, 2005; He, 2009; Ahmad et al., 2010). In Arabidopsis, four classical pathways including photoperiod, gibberellin (GA) signaling, vernalization, and an autonomous pathway, have been identified to regulate flowering (Quesada et al., 2005). Genes of the autonomous pathway promote the floral transition by inhibiting the expression of FLC, a major flowering repressor gene which encodes a MADS box transcription factor (Michaels and Amasino, 1999; Simpson, 2004). The autonomous pathway mutants exhibit an FLC dependent late-flowering phenotype under both long-day and short-day conditions and respond normally to vernalization and gibberellin treatment (Simpson, 2004). Eight autonomous pathway genes have been identified from the early genetic screening for late-flowering mutants; these genes include $L U$ MINIDEPENDENS (LD), FCA, FY, FPA, FLOWERING LOCUS $K$ (FLK), FVE, FLOWERING LOCUS $D(F L D)$ and RELATIVE OF EARLY FLOWERING 6 (REF6) (Lee et al., 1994; Macknight et al., 1997; Schomburg et al., 2001; He et al., 2003; Simpson et al., 2003; Ausin et al., 2004; Lim et al., 2004; Noh et al., 2004; Jiang et al., 2007). Besides these classical members, several genes encoding protein arginine methyltransferases including AtPRMT4a, AtPRMT4b, AtPRMT5 and AtPRMT10, have also been shown to regulate flowering-time through the autonomous pathway (Niu et al., 2007, 2008; Pei et al., 2007; Wang et al., 2007). AtPRMT4a and $A t P R M T 4 b$ redundantly promote flowering by repressing FLC expression (Niu et al., 2008). AtPRMT5 deficiency causes splicing defects in a large number of genes involved in multiple biological processes, including several RNA processing factors that regulate flowering time. The late-flowering phenotype of atprmt5 mutants is an integrative effect of splicing defects of diverse genes in distinct cellular processes rather than an effect on a single target (Pei et al., 2007; Wang et al., 2007; Schmitz et al., 2008; Deng et al., 2010; Hong et al., 2010; Sanchez et al., 2010). Mutation of AtPRMT10 leads to increased FLC transcript levels and hence a late-flowering phenotype (Niu et al., 2007). This phenotype can be rescued by introducing a normal copy of AtPRMT10 (Niu et al., 2007).

In this work, we further characterized the biochemical properties of AtPRMT10 and its function in regulating flowering time. We found that the conserved "VLD" residues in motif I, the "double-E loop" and two previously unreported residues, Arg 54 (R54) and Cys 259 (C259) are all necessary for the enzymatic activity of AtPRMT10. In addition, AtPRMT10 can methylate itself at $\operatorname{Arg} 13$ (R13). However, an Arg 13 to Lys 13 mutation (R13K) of AtPRMT10 does not affect its enzymatic activity. In vivo complementation assays indicated that the enzymatically functional variants of AtPRMT10 complemented the late-flowering phenotype of atprmt10-1, but the enzymatic activity null AtPRMT10 mutant proteins did not. Thus, our results indicate that protein arginine methyltransferase activity of AtPRMT10 is essential for its function in flowering time regulation.

\section{RESULTS}

\section{Residues essential for AtPRMT10's enzymatic activity in vitro}

Although AtPRMT10 is a plant-specific PRMT, it has all the 
conserved motifs possessed by other PRMTs (Fig. 1A). As the first tri-peptide of motif I, "VLD", is essential for the enzymatic activity of both PRMT1 (Wada et al., 2002) and CARM1 (Lee et al., 2002), we made AtPRMT10 mutations with the conserved "VLD" residues changed to "AAA" (VLD-AAA) (Fig. 1A). Recombinant AtPRMT10 VLD-AAA protein tagged with both MBP (maltose-binding protein) and $10 \times$ HIS (histidine) at the amino-terminus was used as enzyme to catalyze methylation of calf thymus histones in vitro. Clear autoradiography signal bands indicating methylation of histone $\mathrm{H} 2 \mathrm{~A}$ and histone $\mathrm{H} 4$ were observed in the lanes with un-mutated AtPRMT10. Also, the signals on substrates were enhanced with increased amount of AtPRMT10 (Fig. 1B). For the lanes representing mutated AtPRMT10 VLD-AAA, no autoradiography signal was detected despite increased amounts of the mutated enzyme (Fig. 1B). The MBP 10×HIS protein is used as the negative control here (Fig. 1B). These methyltransferase activity assays showed that the "VLD-AAA" mutation disrupts the enzymatic activity of AtPRMT10, which reveals the indispensable role of these evolutionarily conserved "VLD" residues for the catalytic function of AtPRMT10.

We then looked into the "double E loop" within the active site (Weiss et al., 2000; Zhang et al., 2000; Zhang and Cheng, 2003). The two glutamates of the "double-E loop", glutamate 143 (E143) and glutamate (E152) are conserved in AtPRMT10 (Fig. 1A). We made single amino acid substitutions of each the glutamate $(E)$ by glutamine $(Q)$, to produce $\mathrm{E} 143 \mathrm{Q}$ and $\mathrm{E} 152 \mathrm{Q}$ versions of AtPRMT10. Methyltransferase activity assays showed that when either E143 or E152 of AtPRMT10 was changed to Q (E143Q or E152Q), the

A

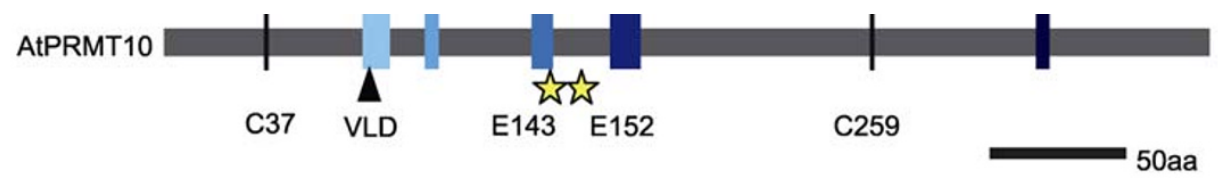

I post I II III THW

B

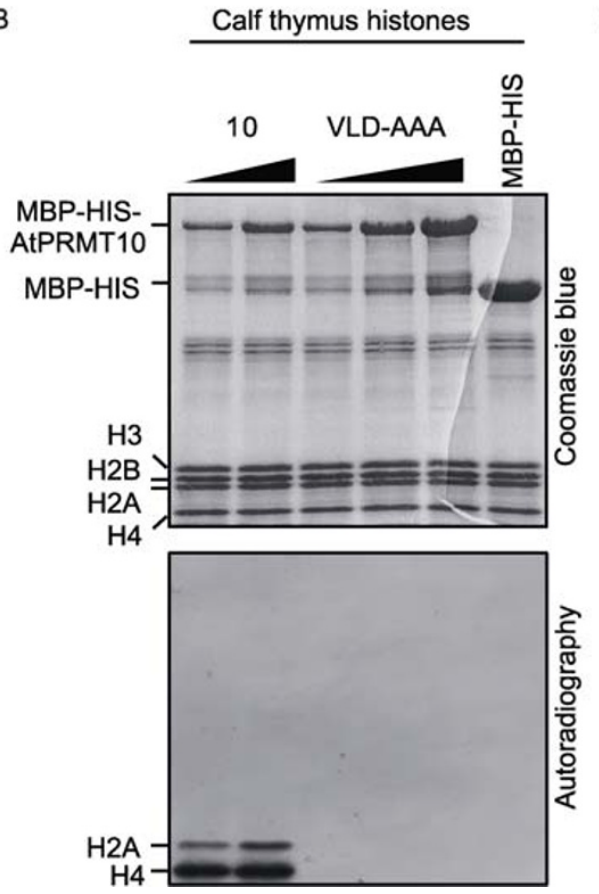

C

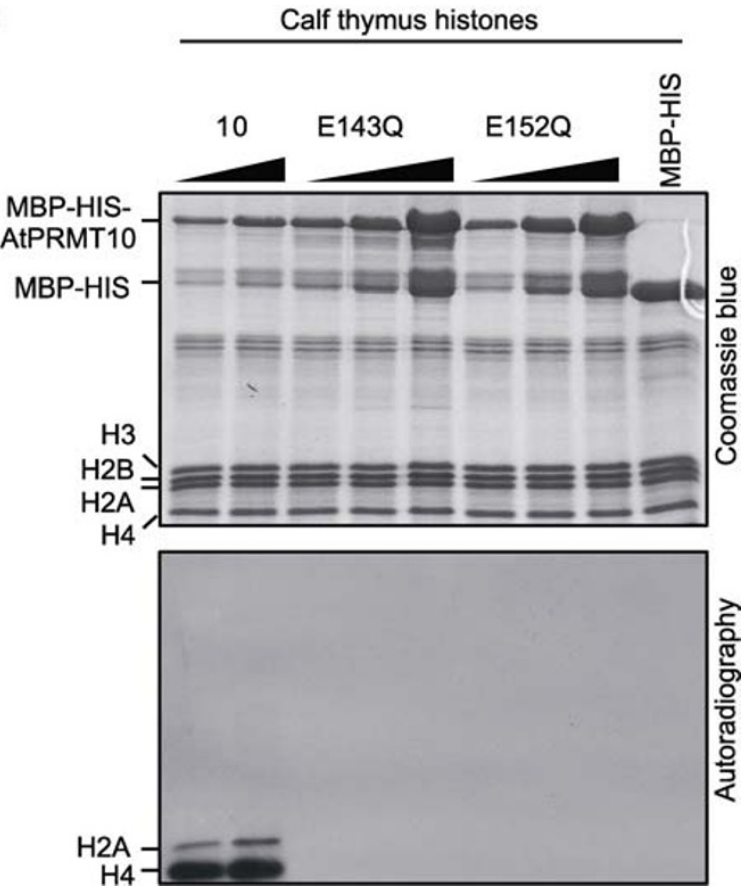

Figure 1. "VLD" residues and "double E" loop are essential for AtPRMT10 methyltransferase activity. (A) The protein structure of AtPRMT10. The structure of the conserved domains of AtPRMT10 is indicated by colored boxes. The "VLD" residues are marked by a triangle. The two conserved glutamates of the "double E" loop are indicated by stars. The verticals point to the positions of the two conserved cysteines. (B) Different amounts of the MBP and HIS double tagged AtPRMT10 VLD-AAA were used in methyltransferase assay against calf thymus histones with radioactive methyl donor S-adenosyl-L-[methyl-3H]Met. (C) Different amounts of the MBP and HIS double tagged AtPRMT10 E143Q and AtPRMT10 E152Q were used in methyltransferase assay against calf thymus histones with radioactive methyl donor S-adenosyl-L-[methyl-3H]Met. The Coomassie blue stained gels are shown on the top and the autoradiographs are shown on the bottom (B and $C)$. 
methylation signal could not be detected (Fig. 1C). This result suggests the importance of these two glutamate residues in enzymatic activity of AtPRMT10.

In addition to those conserved residues situated within the canonical functional motifs of PRMTs, we discovered another novel cysteine residue that was also required for enzymatic activity of AtPRMT10. There are five cysteine residues in AtPRMT10. Among them, only cysteine 259 (C259) and cysteine 37 (C37) are conserved between AtPRMT10 and OsPRMT10 (Niu et al., 2007). We changed each of the two cysteines to serines (C259S or C37S) and found that the C37S did not affect the enzymatic activity of AtPRMT10, but the C259S mutation significantly reduced AtPRMT10 arginine methyltransferase activity compared to the wild-type and the C37S mutated forms of AtPRMT10 (Fig. 2). Referring to the recently resolved AtPRMT10 crystal structure, C259 locates on the outer surface of the C-terminal $\beta$-barrel domain (Cheng et al., 2011). The mechanism by which this C259 residue regulates the methyltransferase activity of AtPRMT10 will be further examined.

\section{Auto-methylation of AtPRMT10 at Arg13}

According to The Arabidopsis Information Resource 10 (TAIR10) database, AtPRMT10 has two different transcripts, At1G04870.1 and At1G04870.2. Through sequence alignment, we found that At1G04870.2 has a 10 nucleotide (nt) extension at the 5 ' end of the second exon, compared to At1G04870.1; this difference may be caused by alternative 3' end splicing in the first intron (Fig. 3A). The At1G04870.1

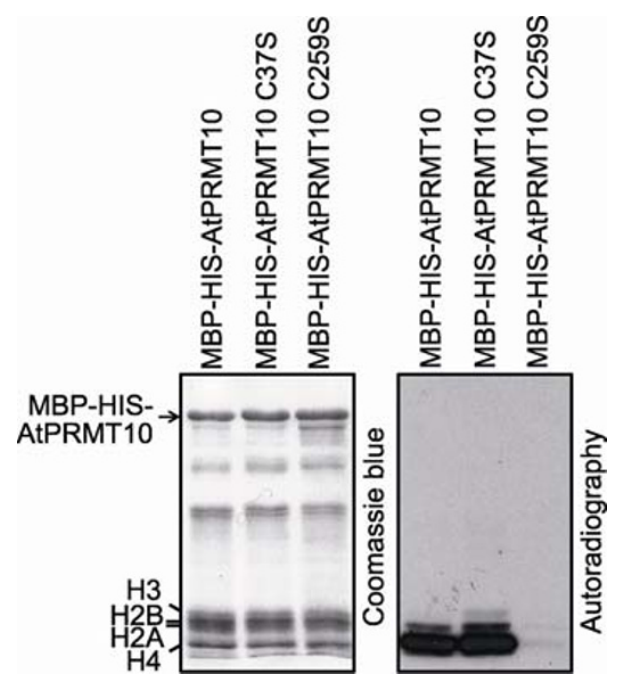

Figure 2. C259S is required for AtPRMT10 methyltransferase activity. Enzymatic activity assay of the MBP and HIS double tagged AtPRMT10, AtPRMT10 C37S and AtPRMT10 C259S against calf thymus histones with radioactive methyl donor S-adenosyl-L-[methyl-3H]Met. Coomassie blue-stained gel is shown on the left and the autoradiographs of the gel is shown on the right. transcript uses the ATG codon inside the second exon as its translational start codon and encodes a peptide of 280 amino acids, while the At1G04870.2 transcript starts translation from the first exon and produces a larger protein with 103 extra amino acids on its $\mathrm{N}$-terminal end (Fig. 3A). To test the enzymatic activities of these predicted proteins, we made constructs to express the proteins in bacteria. Since we could not distinguish the transcript of At1g04870.1 from At1g04870.2 by PCR in plant, we artificially amplified the coding sequence corresponding to the 280-amino acid protein from At1G04870.2 and named it AtPRMT10S. The larger protein translated from At1G04870.2 was named AtPRMT10 (Fig. 3A).

In vitro methyltransferase activity assays showed that only Glutathione-S-Transferase (GST) tagged AtPRMT10 (GSTAtPRMT10), but not GST-AtPRMT10S, could methylate calf thymus histone $\mathrm{H} 2 \mathrm{~A}$ and $\mathrm{H} 4$, which implied that methyltransferase activity was absent in AtPRMT10S (Fig. 3B). We previously observed that AtPRMT10 could also be auto-methylated in the absence of suitable substrates (Niu et al., 2007). When methyltransferase assays were performed with GST tagged AtPRMT10 and AtPRMT10S, we found a single autoradiography band corresponding to the position of AtPRMT10, indicating that the AtPRMT10s could not be methylated by AtPRMT10 (Fig. 3C). GST protein only was used as the negative control. As the AtPRMT10S sequence is identical to the 104-383 amino acids of AtPRMT10, this observation suggests that the auto-methylation site might be within the first 103 amino acids in AtPRMT10.

To identify the auto-methylation site, we used site-directed mutagenesis to alter each of the AtPRMT10 arginine residues and then conducted methylation assays to determine whether the change affected auto-methylation. There are five arginine residues, $\mathrm{R} 2, \mathrm{R} 13, \mathrm{R} 52, \mathrm{R} 54$ and $\mathrm{R} 94$, in the first 103 amino acids of AtPRMT10 (Fig. 3A). We made mutants by changing each of the five arginines to lysines $(K)$. We found that AtPRMT10 R13K and AtPRMT10 R54K did not have automethylation activity (Fig. 3D). However, methylation assays using calf thymus histones as substrates indicated that AtPRMT10 R13K was enzymatically normal, but AtPRMT10 R54K was enzymatically null (Fig. 3E). In accordance with this result, the structure-based sequence alignment of AtPRMT10 with other PRMTs from animals and yeast reveals that the R54 residue is highly conserved and is involved in SAM binding by AtPRMT10 (Cheng et al., 2011). In addition, when mass spectrometry (MS) was used to detect the modification status of GST-AtPRMT10 after auto-methylation reaction, only R13 was also identified as the methylation site (Fig. S1). The above evidence demonstrated that R13 is the single auto-methylation site, and R54 is essential for AtPRMT10 enzymatic activity. It is worth noting that the R13K mutation did not affect AtPRMT10's ability to methylate histones, which suggests that auto-methylation may be unrelated to its histone methyltransferase activity in vitro (Fig. 3E). 

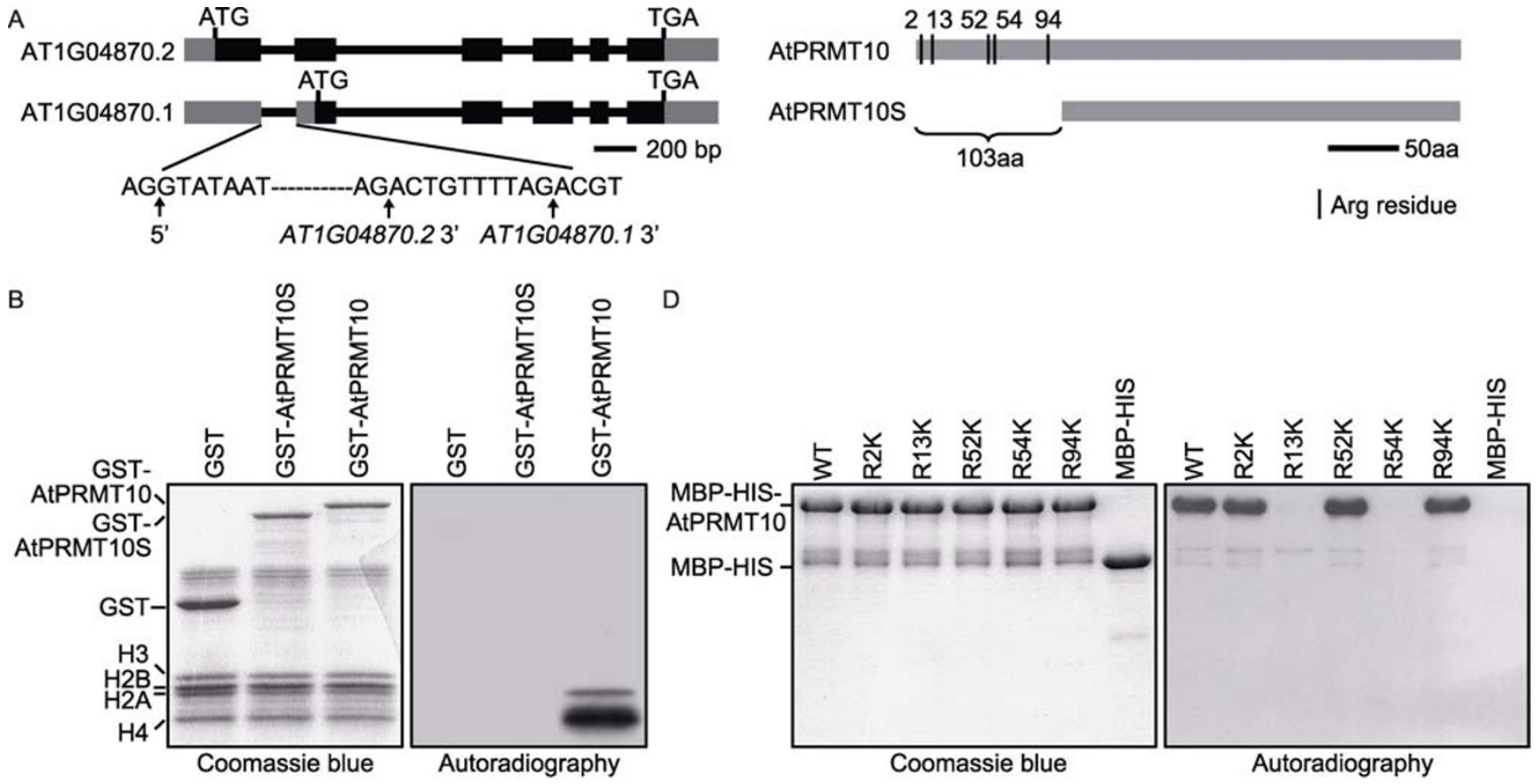

C

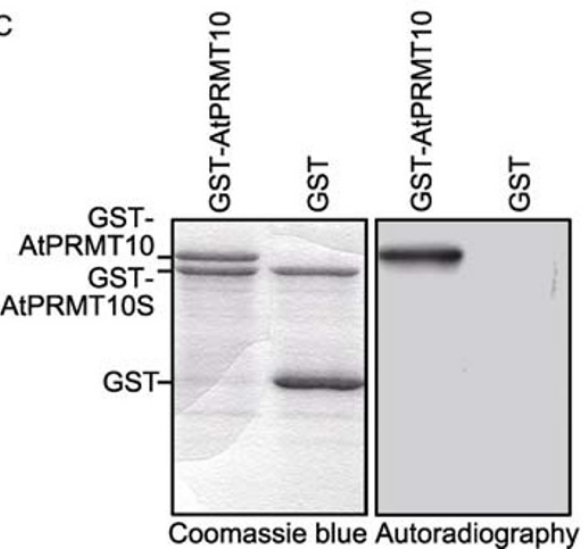

$E$

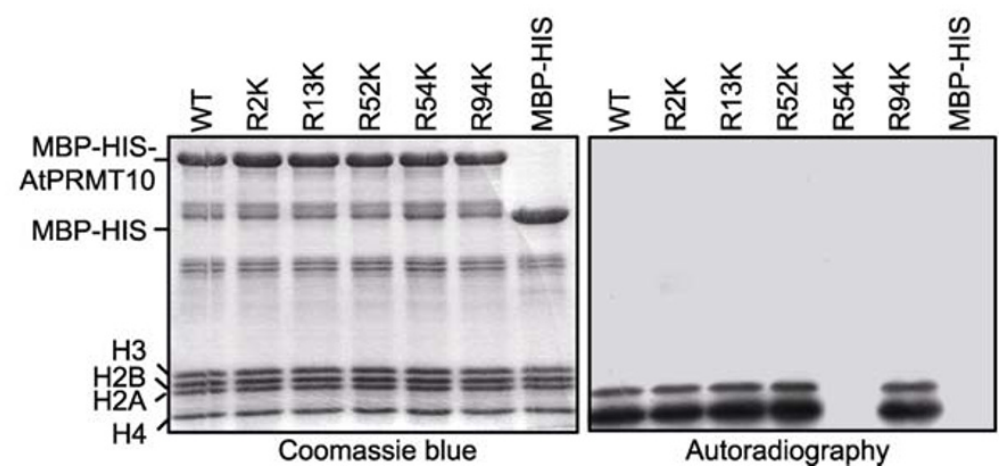

Figure 3. AtPRMT10 auto-methylation at R13. (A) The gene structures of AtPRMT10 and AtPRMT10S are shown on the left. Grey rectangles present UTRs, black rectangles present exons, and the lines show the introns. The nucleotide sequence of the two exon-intron junctions of the first intron is shown, and the alternative splicing sites of the two transcripts are marked by arrows. The protein structures of AtPRMT10 and AtPRMT10S are shown on the right. The extra 103 amino acids of AtPRMT10 compared to AtPRMT10S are marked by braces. The verticals indicate the positions of arginine residues within the first 103 amino acids (aa). (B) Methyltransferase activity assays of GST tagged AtPRMT10 and AtPRMT10S toward calf thymus histones. (C) GST-AtPRMT10 fusion protein was used to react with the GST-AtPRMT10S protein. (D and E) The MBP and HIS double-tagged AtPRMT10 proteins with series single arginine to lysine mutation were used in the methylation assay with $(E)$ and without $(D)$ calf thymus histones, respectively. The Coomassie blue stained gels are shown on the left and the autoradiographs are shown on the right ( $B$ to $E$ ).

\section{AtPRMT10 enzymatic activity is essential for flowering time regulation}

Recent findings demonstrated that some of the biological roles carried out by histone modifying enzymes are independent of their enzymatic activity. For example, LID2 promotes gene silencing in heterochromatin regions but promotes gene activation in euchromatin regions. The role of
LID2 in euchromatin does not require its enzymatic activity, but it does require an intact JmjC domain (Li et al., 2008). Taking advantage of these enzymatic normal or null mutations, we then sought to test if the arginine methyltransferase activity of AtPRMT10 is essential for its biological roles.

Our previous work demonstrated that AtPRMT10 belongs to the autonomous pathway, which regulates flowering time by negatively regulating FLC transcript levels. To test 
whether the AtPRMT10 mutants can function in regulation of flowering time, we first transformed the AtPRMT10 genomic fragment of wild type and the mutant form carrying the $R 13 \mathrm{~K}$ mutation into the atprmt10-1 mutant (Fig. 4). The flowering

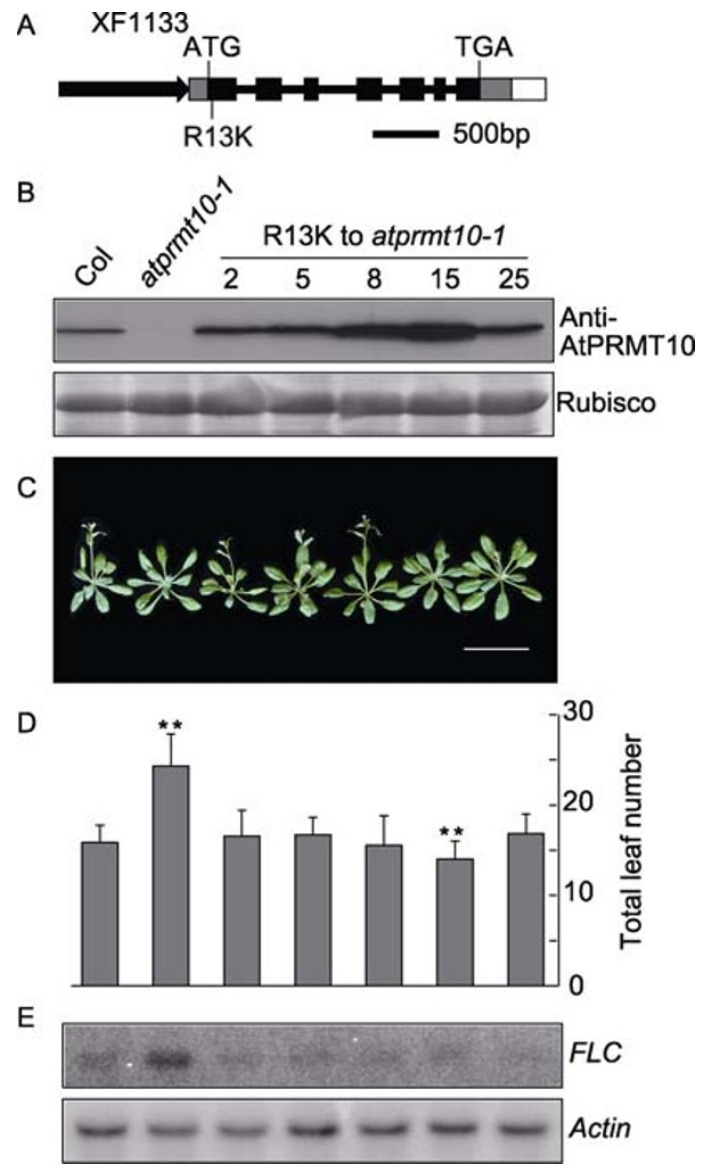

Figure 4. AtPRMT10 $R 13 K$ can rescue the late-flowering phenotype of atprmt10. (A) Diagram of the AtPRMT10 R13K DNA fragment used in complementation assays. The arrow shows the promoter and the box in white represents the 269bp sequence downstream of the 3' UTR. 5' and 3' UTR regions are displayed by grey rectangles. Exons are shown by black rectangles, and the introns are drawn as lines. The mutated arginine residue, translational start codon and termination codon are all marked at the relative positions. (B) Western blot analysis of AtPRMT10 R13K in transgenic lines. Five transgenic lines were analyzed using antiAtPRMT10 (top). The Ponceau S stained Rubisco protein was used as a loading control (bottom). The numbers labeled above indicate different transgenic lines, respectively. Wild-type Col was used as a positive control, whereas atprmt10-1 was used as a negative control. (C) The flowering phenotype of the above plants. The plants shown here were grown under long-day conditions. The white bar represents 5 centimeters. (D) Total leaf number of the above plants when bolted under long-day conditions. Error bars represent $2 \times$ s.e. The statistical significance marked by double stars is determined by the Student's $t$-test at $P<0.01$. The exact $P$ values are supplied in supplemental table 2. (E) RNA gel blot analysis of FLC expression level in the above plants. Actin was used as a loading control. time of the transgenic lines under long day conditions was analyzed by counting the total number of rosette and cauline leaves when the plants bolted. The genomic fragment of wild-type AtPRMT10 complemented the late-flowering phenotype of atprmt10-1 as we showed previously (Niu et al., 2007). The AtPRMT10 R13K transgenic lines we analyzed showed similar AtPRMT10 protein levels as that in the wild-type plants (Fig. 4B). The transgenic lines harboring AtPRMT10 R13K also showed no obvious defects and flowered at a similar time to the wild-type plants (Fig. 4C and 4D). To further confirm the phenotypic observation at molecular level, we tested $F L C$ expression levels in these transgenic lines by northern blot. We found that FLC expression levels in these transgenic lines were similar to the wild-type level, consistent with our phenotypic observation (Fig. 4E). Thus, this data suggest that the methylation of R13 on AtPRMT10 is not required for repression of $F L C$ and flowering time regulation. Given the fact that AtPRMT10 R13K retains methyltransferase activity, this result also suggests a possible connection between enzymatic activity and the flowering time regulation in AtPRMT10.

Similarly, we tested whether "VLD-AAA", E143Q and E152Q substitutions complemented the late-flowering phenotype of atprmt10-1 (Fig. 5). For each transgene harboring mutant forms, five transgenic lines with AtPRMT10 protein levels similar to wild-type Col were used for further analysis (Fig. 5B). We found that all the three types of transgenic plants displayed late-flowering phenotypes similar to that of atprmt10-1, as measured by total leaf number when the plants bolted (Fig. 5C). At the molecular level, the FLC transcripts in these transgenic lines were also similar to that in atprmt10-1 (Fig. 5D). Since the mutant proteins without enzymatic activity could not complement the late-flowering phenotype of atprmt10, therefore, we concluded that arginine methyltransferase activity of AtPRMT10 is essential for its role in repressing of $F L C$ and promoting flowering time in Arabidopsis.

\section{DISSCUSION}

Our previous work showed that the Brassica oleracea Var. botrytis Linn arginine methyltransferase 10 (PHRMT10) likely exists as a dimer (Niu et al., 2007). As PHRMT10 is closely related to AtPRMT10, we analyzed the tag free AtPRMT10 on a Superdex 200 column and identified its dimer form (Fig. $\mathrm{S} 2 \mathrm{~A})$. Interestingly, we found that the C259S mutation partially interfered with the dimerization of AtPRMT10 (Fig. S2B). Conversely, the C37S mutation did not affect the dimerization (Fig. S2B). Dimerization can occur through a disulfide bond between two cysteine residues. However, the crystal structure of AtPRMT10 showed that the C259 residue is positioned on the outer surface of the $\beta$-barrel domain, which is far from the contacting region of the dimer. This obvious steric barrier negates the possibility that $\mathrm{C} 259$ regulates 
A

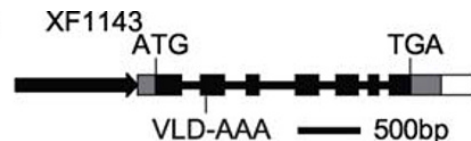

B

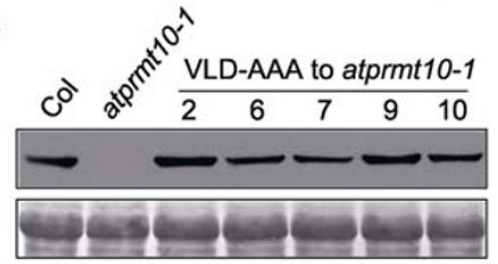

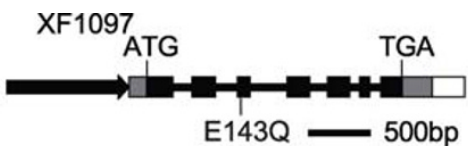

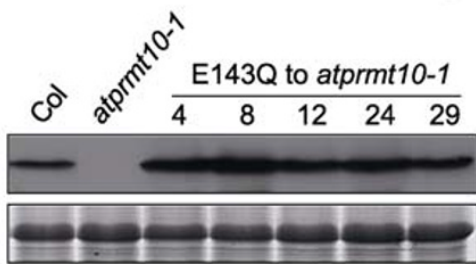

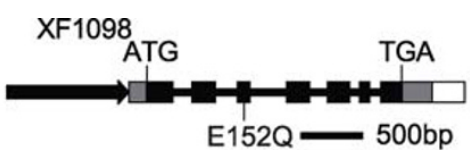

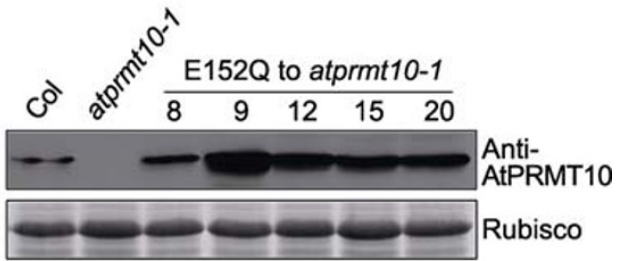

C

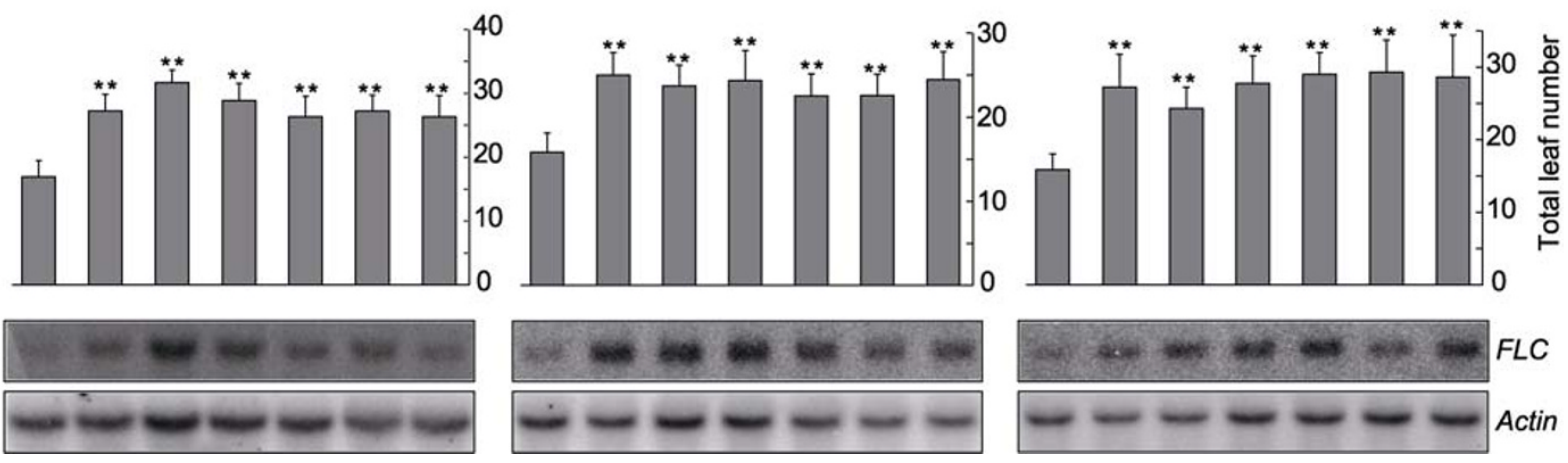

Figure 5. Enzymatic activity null AtPRMT10 cannot rescue the late-flowering phenotype of atprmt10. (A) Diagrams of AtPRMT10 VLD-AAA (left), AtPRMT10 E143Q (middle) and AtPRMT10 E152Q (right) DNA fragments used in complementation assay. The arrow represents the AtPRMT10 promoter, and the box in white represents the 269-bp sequence downstream of the 3' UTR. 5' and 3' UTR regions are displayed by grey rectangles. Exons are shown by black rectangles, and the introns are drawn as lines. The mutated arginine residues, translational start codon and termination codon are all marked at the relative position. (B) Western blot analysis of AtPRMT10 VLD-AAA (left), AtPRMT10 E143Q (middle) and AtPRMT10 E152Q (right) in transgenic lines. Five lines of each transgene were analyzed using anti-AtPRMT10 (top). The Ponceau S stained Rubisco protein was used as a loading control (bottom). The numbers labeled above indicate different transgenic lines, respectively. Wild-type Col was used as a positive control, whereas atprmt10-1 was used as a negative control. (C) Total leaf number of the above plants when bolted under long-day conditions. Error bars represent $2 \times$ s.e. The statistical significance marked by double stars is determined by the Student's t-test at $P<0.01$. The exact $P$ values are supplied in supplemental table 2 . (D) RNA gel blot analysis of FLC expression levels in the above plants. Actin was used as loading controls.

dimerization through formation of a disulfide bond. In addition to forming disulfide bonds, cysteine residues are also subject to S-nitrosylation, a covalent attachment of a nitrogen monoxide group to the thiol side chain of cysteine (Hess et al., 2005). The importance of S-nitrosylation has been widely demonstrated both in animal and in plants. For example, S-nitrosylation of NPR1 at cysteine-156 facilitates its oligomerization and mutation of Cys156 compromises NPR1mediated disease resistance (Tada et al., 2008). Considering the C259S mutation only partially destroyed AtPRMT10 dimer formation, we propose that mutation of C259 might affect the posttranslational modification or configuration of AtPRMT10 through some unknown mechanism.

So far, four mammalian PRMTs, PRMT1, CARM1/PRMT4, PRMT6 and PRMT8, were found to have auto-methylation activity, and the auto-methylated arginines were identified by mass spectrometry (Frankel et al., 2002; Sayegh et al., 2007; Kuhn et al., 2009, 2011; Gui et al., 2011). For functional dissection, CARM1 with a mutation in the auto- methylation site was found to have comparable enzymatic activity to the wild-type CARM1 (Kuhn et al., 2011). Our results showed that AtPRMT10 could be auto-methylated at R13, and the mutant AtPRMT10 with a disrupted auto-methylation site also retained normal catalytic ability in vitro. Moreover, AtPRMT10 R13K could fully rescue the late-flowering defect of the atprmt10-1 mutant. Nevertheless, it is worth noting that the auto-methylation of mouse CARM1 has been shown to link transcription and pre-mRNA splicing (Kuhn et al., 2011). The biological significance of AtPRMT10 auto- methylation remains to be investigated in Arabidopsis.

Through protein sequence alignment, we found that AtPRMT10 shared all the typical motifs found in other PRMTs. Consistent with the results in mammals (Lee et al., 2002; Wada et al., 2002; Zhang and Cheng, 2003; Xu et al., 2004; Higashimoto et al., 2007), the motif I and "double E" loop were indispensable for AtPRMT10 enzymatic activity in vitro. In addition, all enzymatic null AtPRMT10 proteins, including the VLD-AAA, E143Q and E152Q mutant versions, were unable to carry out the biological function of AtPRMT10 in promoting flowering. We also found that the AtPRMT10 
C259S with the impaired enzymatic activity failed to fully rescue the late-flowering phenotype of the atprmt10 mutant (Fig. S3). The close connection between AtPRMT10 methyltransferase activity and its role in flowering time control provides us a clue to further understand its molecular mechanisms in vivo by analyzing its substrates. Identification of its substrate involved in flowering time regulation will give us a better understanding of how protein arginine methylation is involved in repressing FLC expression and promoting flowering in Arabidopsis.

\section{MATERIALS AND METHODS}

\section{Plasmid Construction}

For the protein expression plasmids, the AtPRMT10S cDNA was amplified from AtPRMT10 in the expression vector XF272 (pGEX-4T-2-AtPRMT10) (Niu et al., 2007) and cloned into the pGEX-4T-2 BamHI site. Full length AtPRMT10 cDNA was cloned into the vector XF245 (a modified MBP tag containing plasmid) to create an MBP and $10 \times \mathrm{HIS}$ tagged AtPRMT10 protein with a TEV protease recognition site between the target protein and the MBP and 10×HIS tags. The series of amino acid substitutions were made by modification of these recombinant expression vectors. For the mutant variants of AtPRMT10 complementation constructs, the equivalent site-specific mutations were made on the backbone of the AtPRMT10 complementation vector XF378 (pCAMBIA1300-AtPRMT10) (Niu et al., 2007). Construction of the various amino acid substitution mutants was carried out by a site-directed mutagenesis method using the QuikChange ${ }^{\circledR}$ Site-Directed Mutagenesis Kit (Stratagene) and the mutant plasmids were confirmed by sequencing. Primers used for constructions are described in table S1.

\section{AtPRMT10 dimers assayed by Superdex 200 column}

The recombinant proteins were expressed in Escherichia coli strain $\mathrm{BL} 21$. The MBP and $10 \times \mathrm{HIS}$ tags were removed by digestion with TEV protease followed by affinity resin and their removal was confirmed by SDS-PAGE. The tag-free proteins were analyzed on a Superde $\mathrm{x} 200$ column (GE Healthcare). The column was run in $1 \mathrm{x}$ PBS buffer with AKTA purifier system (GE Healthcare). A standard sample of known molecular weight was used to calibrate the Superde $x$ 200 column.

Plant materials, growth conditions and flowering time assessment

Experiments were performed using Arabidopsis (Arabidopsis thaliana) accession Col-0. The mutant line, atprmt10-1 (SALK_047046) (Niu et al., 2007), from the SALK collection was supplied by the Arabidopsis Biological Resource Center (Ohio State University).

Plant growth, flowering time analysis, and plant transformation were performed as reported previously (Niu et al., 2007; Pei et al., 2007). To quantify flowering time, the total number of rosette and cauline leaves were counted when the plants bolted. At least 15 plants were analyzed for each measurement.

\section{Complementation assay}

The AtPRMT10 complementation construct (Niu et al., 2007) and its mutant variants were transformed into Agrobacterium strain GV3101 and further transformed into atprmt10-1 through floral dipping (Bechtold and Pelletier, 1998). T1 generation seeds were selected on MS plates (Zhang et al., 2011a) containing $25 \mu \mathrm{g} / \mathrm{mL}$ hygromycin. The F2 progenies selected by hygromycin were used for RNA gel blot analysis to detect FLC expression levels. AtPRMT10 protein levels were analyzed by standard immunoblot with total proteins from 12-day-old seedlings.

\section{Protein arginine methyltransferase assay}

Recombinant proteins were expressed in Escherichia coli strain BL21, and methyltransferase activity assays were performed as described (Niu et al., 2007; Pei et al., 2007). Calf thymus histones were purchased from Roche.

\section{Western blot assay}

Total proteins were extracted from 12-day-old wild-type Col, atprmt10-1 and transgenic plants using buffer A $(50 \mathrm{mmol} / \mathrm{L}$ Tris- $\mathrm{HCl}$ (pH 7.4), $0.5 \mathrm{mmol} / \mathrm{L}$ EDTA, $20 \mathrm{mmol} / \mathrm{L}\left(\mathrm{NH}_{4}\right)_{2} \mathrm{SO}_{4}, 25 \%$ [v/v] glycerol, $1 \mathrm{mmol} / \mathrm{L}$ PMSF, $0.5 \mathrm{mmol} / \mathrm{L} \mathrm{DTT})$. The extracts were separated by $10 \%$ SDS-PAGE and transferred to Hybond ECL membrane (GE Healthcare). A mouse polyclonal antibody specific to AtPRMT10 was used for immunoblotting (Niu et al., 2007). Supersignal West Dura substrate (Hwang et al., 2008) was used to detect the HRP-conjugated secondary antibodies. The membranes stained with Ponceau $\mathrm{S}$ were used as loading controls.

\section{Northern blot assays}

Total RNAs were extracted from 12-day-old wild-type Col, atprmt10-1 and transgenic plants using the Trizol reagent (Invitrogen) according to the manufacturer's instructions. The concentration of each sample was measured by NanoDrop ND-1000 (Thermo Scientific). About 15 $\mu \mathrm{g}$ total RNA from each sample was separated on $1.2 \%$ agarose-formaldehyde gel, and then transferred onto Hybond-XL nylon membrane (GE Healthcare). ${ }^{32} \mathrm{P}$-labeled single strand DNA complementary to FLC mRNA was used as probe. Primers used for FLC full length cDNA probe template amplification were cx461 (5'-CGCGGATCCATGGGAAGAAAAAAACTAGA-3') and cx462 (5'-CCGGAATTCCTAATTAAGTAGTGGGAGAGT-3'). The Actin gene was used as a loading control.

\section{ACKNOWLEDGEMENTS}

We would like to thank Aiying Zhang and Qingbao Zhu in Xiaofeng Cao's lab for technical supports. We also thank The Arabidopsis Biological Resource Center at Ohio State for providing SALK T-DNA-insertion lines. This work was supported by the National Basic Research Program of China (Nos. 2011CB915400 and 2009CB941500 to X.C.), the National Natural Science Foundation of China (Grant Nos. 30930048 and 30921061 to X.C., and 90919033 
to C.L.), and the Chinese Academy of Sciences (No. KSCX2-EWQ-24-02 to C.L.)

\section{ABBREVIATIONS}

ADMA, asymmetric di-methylarginine; CARM1, coactivator associated arginine methyltransferase; FLC, flowering locus C; FLD, flowering locus D; FLK, flowering locus K; GST, glutathioneS-transferase; HIS, histidine; LD, luminidependens; MBP, maltose Binding Protein; MMA, monomethylarginine; MS, mass spectrometry; PRMT, protein arginine methyltransferase; REF6, relative of early flowering 6; SAM, S-adenosyl-L-methionine; SDMA, symmetric di-methylarginine; SDS-PAGE, sodium-dodecyl-sulphate polyacrylamide gel electrophores

\section{REFERENCES}

Ahmad, A., and Cao, X. (2012). Plant PRMTs broaden the scope of arginine methylation. J Genet Genomics (In Press).

Ahmad, A., Zhang, Y., and Cao, X.F. (2010). Decoding the epigenetic language of plant development. Mol Plant 3, 719-728.

Amasino, R.M. (2005). Vernalization and flowering time. Curr Opin Biotechnol 16, 154-158.

Ausin, I., Alonso-Blanco, C., Jarillo, J.A., Ruiz-Garcia, L., and Martinez-Zapater, J.M. (2004). Regulation of flowering time by FVE, a retinoblastoma-associated protein. Nat Genet 36, 162-166.

Bechtold, N., and Pelletier, G. (1998). In planta agrobacterium-mediated transformation of adult Arabidopsis thaliana plants by vacuum infiltration. Arabidopsis Protoc 82 , 259-266.

Bedford, M.T., and Clarke, S.G. (2009). Protein arginine methylation in mammals: who, what, and why. Mol Cell 33, 1-13.

Cheng, X., Collins, R., and Zhang, X. (2005). Structural and sequence motifs of protein (histone) methylation enzymes. Annu Rev Biophys Biomol Struct 34, 267-294.

Cheng, Y., Frazier, M., Lu, F., Cao, X., and Redinbo, M.R. (2011). Crystal structure of the plant epigenetic protein arginine methyltransferase 10. J Mol Biol 414, 106-122.

Deng, X., Gu, L., Liu, C., Lu, T., Lu, F., Lu, Z., Cui, P., Pei, Y., Wang, B., Hu, S., et al. (2010). Arginine methylation mediated by the Arabidopsis homolog of PRMT5 is essential for proper pre-mRNA splicing. Proc Natl Acad Sci U S A 107, 19114-19119.

Frankel, A., Yadav, N., Lee, J., Branscombe, T.L., Clarke, S., and Bedford, M.T. (2002). The novel human protein arginine $\mathrm{N}$-methyltransferase PRMT6 is a nuclear enzyme displaying unique substrate specificity. J Biol Chem 277, 3537-3543.

Gui, S., Wooderchak, W.L., Daly, M.P., Porter, P.J., Johnson, S.J., and Hevel, J.M. (2011). Investigation of the molecular origins of protein-arginine methyltransferase I (PRMT1) product specificity reveals a role for two conserved methionine residues. J Biol Chem 286, 29118-29126.

$\mathrm{He}, \mathrm{Y}$. (2009). Control of the transition to flowering by chromatin modifications. Mol Plant 2, 554-564.

Herrmann, F., and Fackelmayer, F. (2009). Nucleo-cytoplasmic shuttling of protein arginine methyltransferase 1 (PRMT1) requires enzymatic activity. Genes Cells 14, 309-317.
Hess, D.T., Matsumoto, A., Kim, S.O., Marshall, H.E., and Stamler, J.S. (2005). Protein S-nitrosylation: purview and parameters. $\mathrm{N}$ at Rev Mol Cell Biol 6, 150-166.

Higashimoto, K., Kuhn, P., Desai, D., Cheng, X., and Xu, W. (2007). Phosphorylation-mediated inactivation of coactivator-associated arginine methyltransferase 1. Proc Natl Acad Sci U S A 104, 12318-12323.

Hong, S., Song, H.R., Lutz, K., Kerstetter, R.A., Michael, T.P., and McClung, C.R. (2010). Type II protein arginine methyltransferase 5 (PRMT5) is required for circadian period determination in Arabidopsis thaliana. Proc Natl Acad Sci U S A 107, 21211-21216.

Hwang, H., Pierce, B., Mintseris, J., Janin, J., and Weng, Z. (2008). Protein-protein docking benchmark version 3.0. Proteins 73 , 705-709.

Jiang, D., Yang, W., He, Y., and Amasino, R.M. (2007). Arabidopsis relatives of the human lysine-specific demethylase1 repress the expression of FWA and FLOWERING LOCUS C and thus promote the floral transition. Plant Cell 19, 2975-2987.

Kuhn, P., Chumanov, R., Wang, Y., Ge, Y., Burgess, R.R., and Xu, W. (2011). Automethylation of CARM1 allows coupling of transcription and mRNA splicing. Nucleic Acids Res 39, 2717-2726.

Kuhn, P., Xu, Q., Cline, E., Zhang, D., Ge, Y., and Xu, W. (2009). Delineating Anopheles gambiae coactivator associated arginine methyltransferase 1 automethylation using top-down high resolution tandem mass spectrometry. Protein Sci 18, 1272-1280.

Kwak, Y.T., Guo, J., Prajapati, S., Park, K.J., Surabhi, R.M., Miller, B., Gehrig, P., and Gaynor, R.B. (2003). Methylation of SPT5 regulates its interaction with RNA polymerase II and transcriptional elongation properties. Mol Cell 11, 1055-1066.

Lee, I., Aukerman, M., Gore, S., Lohman, K., Michaels, S., Weaver, L., John, M., Feldmann, K., and Amasino, R. (1994). Isolation of LUMINIDEPENDENS: a gene involved in the control of flowering time in Arabidopsis. The Plant Cell 6, 75-83.

Lee, Y.H., Koh, S.S., Zhang, X., Cheng, X., and Stallcup, M.R. (2002). Synergy among nuclear receptor coactivators: selective requirement for protein methyltransferase and acetyltransferase activities. Mol Cell Biol 22, 3621-3632.

Li, F., Huarte, M., Zaratiegui, M., Vaughn, M.W., Shi, Y., Martienssen, R., and Cande, W.Z. (2008). Lid2 is required for coordinating $\mathrm{H} 3 \mathrm{~K} 4$ and $\mathrm{H} 3 \mathrm{~K} 9$ methylation of heterochromatin and euchromatin. Cell 135, 272-283.

Lim, M.H., Kim, J., Kim, Y.S., Chung, K.S., Seo, Y.H., Lee, I., Hong, C.B., Kim, H.J., and Park, C.M. (2004). A new Arabidopsis gene, FLK, encodes an RNA binding protein with $\mathrm{K}$ homology motifs and regulates flowering time via FLOWERING LOCUS C. Plant Cell 16, 731-740.

Liu, C., Lu, F., Cui, X., and Cao, X. (2010). Histone methylation in higher plants. Annu Rev Plant Biol 61, 395-420.

Macknight, R., Bancroft, I., Page, T., Lister, C., Schmidt, R., Love, K., Westphal, L., Murphy, G., Sherson, S., and Cobbett, C. (1997). FCA, a gene controlling flowering time in Arabidopsis, encodes a protein containing RNA-binding domains. Cell 89, 737-745.

Michaels, S.D., and Amasino, R.M. (1999). FLOWERING LOCUS C encodes a novel MADS domain protein that acts as a repressor of 
flowering. Plant Cell 11, 949-956.

Niu, L., Lu, F., Pei, Y., Liu, C., and Cao, X. (2007). Regulation of flowering time by the protein arginine methyltransferase AtPRMT10. EMBO Rep 8, 1190-1195.

Niu, L., Zhang, Y., Pei, Y., Liu, C., and Cao, X. (2008). Redundant requirement for a pair of PROTEIN ARGININE METHYLTRANSFERASE4 homologs for the proper regulation of Arabidopsis flowering time. Plant Physiol 148, 490-503.

Noh, B., Lee, S.H., Kim, H.J., Yi, G., Shin, E.A., Lee, M., Jung, K.J., Doyle, M.R., Amasino, R.M., and Noh, Y.S. (2004). Divergent roles of a pair of homologous jumonji/zinc-finger-class transcription factor proteins in the regulation of Arabidopsis flowering time. Plant Cell 16, 2601-2613.

Pei, Y., Niu, L., Lu, F., Liu, C., Zhai, J., Kong, X., and Cao, X. (2007). Mutations in the Type II protein arginine methyltransferase AtPRMT5 result in pleiotropic developmental defects in Arabidopsis. Plant Physiol 144, 1913-1923.

Quesada, V., Dean, C., and Simpson, G.G. (2005). Regulated RNA processing in the control of Arabidopsis flowering. Int $\mathrm{J}$ Dev Biol 49, 773-780.

Sanchez, S.E., Petrillo, E., Beckwith, E.J., Zhang, X., Rugnone, M.L., Hernando, C.E., Cuevas, J.C., Godoy Herz, M.A., Depetris-Chauvin, A., Simpson, C.G., et al. (2010). A methyl transferase links the circadian clock to the regulation of alternative splicing. Nature 468, 112-116.

Sayegh, J., Webb, K., Cheng, D., Bedford, M.T., and Clarke, S.G. (2007). Regulation of protein arginine methyltransferase 8 (PRMT8) activity by its N-terminal domain. J Biol Chem 282, 36444-36453.

Schmitz, R.J., Sung, S., and Amasino, R.M. (2008). Histone arginine methylation is required for vernalization-induced epigenetic silencing of FLC in winter-annual Arabidopsis thaliana. Proc Natl Acad Sci U S A 105, 411-416.

Schluckebier, G., O'Gara, M., Saenger, W., and Cheng, X. (1995). Universal catalytic domain structure of AdoMet-dependent methyltransferases. J Mol Biol 247, 16-20.

Schomburg, F.M., Patton, D.A., Meinke, D.W., and Amasino, R.M. (2001). FPA, a gene involved in floral induction in Arabidopsis, encodes a protein containing RNA-recognition motifs. Plant Cell 13, 1427-1436.

Simpson, G.G. (2004). The autonomous pathway: epigenetic and post-transcriptional gene regulation in the control of Arabidopsis flowering time. Curr Opin Plant Biol 7, 570-574.

Simpson, G.G., Dijkwel, P.P., Quesada, V., Henderson, I., and Dean,
C. (2003). FY is an RNA 3' end-processing factor that interacts with FCA to control the Arabidopsis floral transition. Cell 113, 777-787.

Tada, Y., Spoel, S.H., Pajerowska-Mukhtar, K., Mou, Z., Song, J., Wang, C., Zuo, J., and Dong, X. (2008). Plant immunity requires conformational charges of NPR1 via S-nitrosylation and thioredoxins. Science 321, 952-956.

Wada, K., Inoue, K., and Hagiwara, M. (2002). Identification of methylated proteins by protein arginine $\mathrm{N}$-methyltransferase 1 , PRMT1, with a new expression cloning strategy. Biochim Biophys Acta 1591, 1-10.

Wang, X., Zhang, Y., Ma, Q., Zhang, Z., Xue, Y., Bao, S., and Chong K. (2007). SKB1-mediated symmetric dimethylation of histone H4R3 controls flowering time in Arabidopsis. EMBO J 26, 1934-1941.

Weiss, V., McBride, A., Soriano, M., Filman, D., Silver, P., and Hogle, J. (2000). The structure and oligomerization of the yeast arginine methyltransferase, Hmt1. Nat Struct Biol 7, 1165-1171.

Wolf, S.S. (2009). The protein arginine methyltransferase family: an update about function, new perspectives and the physiological role in humans. Cell Mol Life Sci 66, 2109-2121.

Xu, W., Cho, H., Kadam, S., Banayo, E.M., Anderson, S., Yates, J.R., Emerson, B.M., and Evans, R.M. (2004). A methylation-mediator complex in hormone signaling. Genes Dev 18, 144-156.

Yan, D., Zhang, Y., Niu, L., Yuan, Y., and Cao, X. (2007). Identification and characterization of two closely related histone $\mathrm{H} 4$ arginine 3 methyltransferases in Arabidopsis thaliana. Biochem J 408, 113-121.

Zhang, J., Teng, C., and Liang, Y. (2011a). Programmed cell death may act as a surveillance mechanism to safeguard male gametophyte development in Arabidopsis. Protein Cell 2, 837-844.

Zhang, X., and Cheng, X. (2003). Structure of the predominant protein arginine methyltransferase PRMT1 and analysis of its binding to substrate peptides. Structure 11, 509-520.

Zhang, X., Zhou, L., and Cheng, X. (2000). Crystal structure of the conserved core of protein arginine methyltransferase PRMT3. EMBO J 19, 3509-3519.

Zhang, Z., Zhang, S., Zhang, Y., Wang, X., Li, D., Li, Q., Yue, M., Zhang, Y.E., Xu, Y., Xue, Y., et al. (2011b). Arabidopsis floral initiator SKB1 confers high salt tolerance by regulating transcription and pre-mRNA splicing through altering histone H4R3 and small nuclear ribonucleoprotein LSM4 methylation. Plant Cell 23, 396-411. 